\title{
Frequency of the MDRI mutant allele associated with multidrug sensitivity in dogs from Brazil
}

This article was published in the following Dove Press journal:

Veterinary Medicine: Research and Reports

13 April 2015

Number of times this article has been viewed

\section{Marina M Monobe' \\ João P Araujo Junior ${ }^{2}$ \\ Kari $\bigvee$ Lunsford $^{3}$ \\ Rodrigo C Silva ${ }^{4}$ \\ Camilo Bulla ${ }^{4}$}

'Department of Veterinary Clinics, School of Veterinary Medicine and Animal Science, ${ }^{2}$ Department of Microbiology and Immunology, Biosciences Institute, Sao Paulo State University (UNESP), Botucatu, Brazil; ${ }^{3}$ Department of Clinical Sciences and Animal Health Center, ${ }^{4}$ Department of Pathobiology and Population Medicine, College of Veterinary Medicine, Mississippi State University, Mississippi, MS, USA
Correspondence: Camilo Bulla Department of Pathobiology and Population Medicine, College of Veterinary Medicine, Mississippi State University, 640 Wise Center Drive, Mississippi, MS 39762-6100, USA

Tel + I 662325 I I89

Fax + I 6623254548

Email bulla@cvm.msstate.edu
Abstract: To date, a 4-bp deletion in the MDR1 gene has been detected in more than ten dog breeds, as well as in mixed breed dogs, in several countries, however information regarding this mutation in dogs from Brazil is lacking. For this reason, 103 Collies, 77 Border Collies, 76 Shetland Sheepdogs, 20 Old English Sheepdogs, 55 German Shepherds, 16 Australian Shepherds, and 53 Whippets from Brazil were screened for the presence of the mutation. The heterozygous mutated genotype, MDR1 (+/-), frequency found for Collies, Australian Shepherd, and Shetland Sheepdog was 50.5\% (95\% CI $=41.1 \%-59.9 \%), 31.3 \%(95 \% \mathrm{CI}=8.6 \%-53.2 \%)$, and $15.8 \%(95 \% \mathrm{CI}=7.7 \%-23.9 \%)$, respectively. Homozygous mutated genotype, MDR1 $(-/-)$, was detected only in Collies $35.9 \%$. The MDR1 allele mutant frequency found for Collies, Australian Shepherd, and Shetland Sheepdog was 61.2\% (95\% CI =54.8\%-67.5\%), 15.6\% $(95 \% \mathrm{CI}=3.1 \%-28.2 \%)$, and $7.9 \%(95 \% \mathrm{CI}=3.7 \%-12.1 \%)$, respectively. Additionally, even free of the mutant allele, the maximum mutant prevalence (MMP) in that population, with $95 \%$ CI, was 3.8\%, 5.2\%, 5.4\%, and 13.8\% for Border Collies, German Shepherds, Whippets, and Old English Sheepdogs, respectively. In this way, this information is important, not only for MDR1 genotype-based breeding programs and international exchange of breeding animals of predisposed breeds, but also for modification of drug therapy for breeds at risk.

Keywords: P-glycoprotein, MDR1 mutation, ivermectin, dog, drug sensitivity

\section{Introduction}

Encoded by the MDR1 (ABCB1) gene, the multidrug resistance (MDR) transporter P-glycoprotein (P-gp) belongs to the family of membrane-bound ATP-binding cassette (ABC) transporters. ${ }^{1}$ It is expressed in many tissues (with secretory or excretory functions) such as the liver (canalicular membrane of hepatocytes), kidney (luminal membrane of proximal tubules), and intestine (brush border membrane of enterocytes), where it limits drug absorption from the gut and promotes drug excretion into the bile and urine. Additionally, P-gp is expressed at physiological barriers, such as the bloodbrain barrier, placenta (trophoblasts), and Sertoli cell barrier, where it restricts drug entry into the central nervous system, fetal circulation, and gonads, respectively. ${ }^{2-4}$

In 2001, ivermectin-sensitive Collies were shown to carry the MDR1 nt230 (del4) mutation (syn MDR1-1Delta, ABCB1-1Delta). ${ }^{5}$ This mutation results in a frameshift deletion that generates multiple premature stop codons, producing a truncated P-gp containing less than $10 \%$ of the wild type amino acid sequence ${ }^{6}$ that likely results in complete loss of P-gp function. The MDR1 nt230 (del4) is conserved in affected dogs and is identical by descent, highlighting the importance of a genotype-based breeding program to eliminate the mutation from genetic pools. ${ }^{7}$ Dogs that are homozygous 
for the mutation have a higher predisposition to intoxication by P-gp substrates than heterozygous dogs, that in turn are more susceptible than homozygous wild-type dogs. ${ }^{8}$ After the first identification of the canine MDR1 mutation in Collies, several molecular techniques have been developed to identify carriers.

Recently, genetic screening of dog breeds has shown that several breeds other than Collies are also affected by the mutation. These breeds include those related to the Collie, such as Australian Shepherds, Border Collies, English shepherds, McNabs, Old English Sheepdogs, Shetland Sheepdogs, and Wällers; ${ }^{7,9}$ and those unrelated to the Collie including German Shepherds, Whippets, Silken Windhounds, and White Swiss Shepherds. Mixed breeds have also been identified with the mutation. ${ }^{10-12}$

To date, there have been no surveys of breed distribution and frequency of this particular mutation in dogs from Brazil. This information is not only important for MDR1 genotypebased breeding programs, including international exchange of stocks of predisposed breeds, but also for improved medical care for individual dogs that may be at risk for the P-gp dysfunction.

\section{Materials and methods}

\section{Animals}

A total number of the Brazilian canine population for each specific breed in the last 10 years $(2,220$ Collies, 4,430 Shetland Sheepdogs, 18,720 Border Collies, 14,438 German Shepherds, 690 Old English Sheepdogs, 4,000 Australian Shepherds, and 3,880 Whippets) was considered according to the registered data in Brazilian Confederation of Kennel Clubs (CBKC, 2011, personal communication). Samples from 103 Collies, 76 Shetland Sheepdogs, 77 Border Collies, 55 German Shepherds (brown colored genotype),
20 Old English Sheepdogs, 16 Australian Shepherds, and 53 Whippets were obtained with owner's consent. In Brazil, Longhaired Whippets and Shorthaired Whippets are considered only one breed by the CBKC, and since we don't have any way to distinguish both breeds, we considered just Whippets for this study. The study was approved by the institutional animal care and use committee.

There was no prior selection of the participating dog population and, to our knowledge, they had not been tested previously for MDR1 mutation. Pedigree (representing the four most recent generations) was available for only 181 dogs.

\section{Sampling}

Samples consisted of buccal mucosa swabs or $5 \mathrm{~mL}$ of whole blood collected from the jugular vein using a Vacutainer collection system with EDTA.

\section{MDRI genotyping}

DNA was extracted from $330 \mu \mathrm{L}$ of blood or from buccal swabs using the Illustra Blood Genomic Prep Mini Spin Kit (GE Healthcare, Piscataway, NJ, USA) following the manufacturer's recommended protocol. The DNA was stored at $-80^{\circ} \mathrm{C}$ until $\mathrm{PCR}$ (polymerase chain reaction) analysis was performed. For MDR1 genotype, an allele-specific PCRbased screening method was applied to detect the mutant allele and to determine if a dog is homozygous or heterozygous for the mutation (Figure 1). As positive control, DNA from six dogs (Collies) that were carriers of the mutation, identified by direct DNA sequence analysis carried out to confirm the sequence of MDR1 (+/-), was used. The reaction was considered valid when a PCR product with approximately 480 base pairs (bp) was amplified with the primers P-gpA and P-gpD, as previously described. ${ }^{13}$

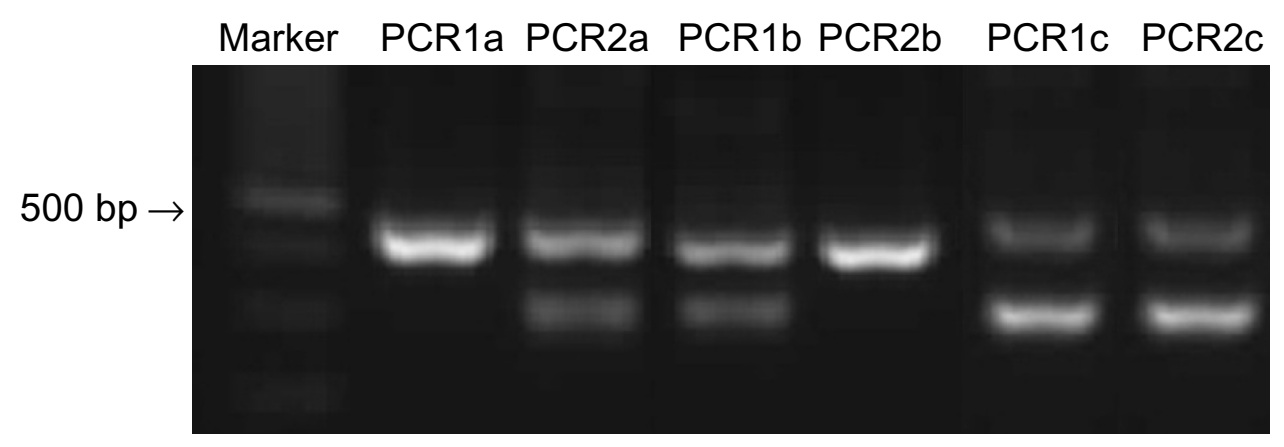

Figure I Results of allele-specific PCR testing from dogs with different MDRI genotypes.

Notes: Homozygous for the MDR I mutant allele [MDRI (-/-)]; homozygous for the MDR I wild type allele [MDR I (+/+)]; heterozygous for the MDR I mutant allele [MDR I $(+/-)$ ]. Lane I: Molecular weight 100 bp DNA ladder (Life Technologies, Waltham, MA, USA). The internal control 483 or 479 bp amplicons were presented in all samples for both PCR reactions (lanes 2-7). PCRI presented an extra 326 bp amplicon in samples from dogs with two (lane 4) or one (lane 6) MDRI wild type alleles. In PCR2 the extra 326 bp amplicon was presented in samples from dogs with two (lane 3) or one (lane 7) MDRI mutant alleles.

Abbreviation: PCR, polymerase chain reaction. 


\section{Sequencing}

The primers P-gpA and P-gpD were used to amplify a segment of the MDR1 gene spanning the mutation site. ${ }^{13} \mathrm{PCR}$ products were purified using QIAquick PCR Purification Kit (Qiagen ${ }^{\circledR}$, Valencia, CA, USA) and were directly sequenced using BigDye ${ }^{\circledR}$ Terminator v3.1 Cycle Sequencing Kit and Applied Biosystems 3500 Genetic Analyzers (Waltham, MA, USA) sequencer. Sequencing analysis of the amplified material identified six dogs (Collies) that were carriers of the mutation, which were then used as the positive control for PCR1 and PCR2 reactions.

\section{Data analysis}

The results were tabulated as dichotomous variables (presence or absence of mutation) as well as homozygous wild type [MDR1 $(+/+)]$, heterozygous [MDR1 $(+/-)$ ], or homozygous mutant [MDR1 (-/-)], for subsequent analysis. The frequency, as well as the $95 \%$ confidence interval $(95 \% \mathrm{CI})$ to determine the power, were calculated for each genotype [MDR1 (+/+), MDR1 (+/-), MDR1 (-/-)] and for allele mutation [zero, one and two alleles mutations counted for MDR1 (+/+), MDR1 (+/-), MDR1 (-/-) respectively]. For those breeds found free of the mutant alleles, the probabilities of the maximum mutation prevalence (MMP) with 95\% CI, were calculated based on the significance level $(\alpha=0.05)$, the number of animals tested (n), and the total number of animals in the population of each breed $(\mathrm{N})$ with the following formula, adapted from Cannon et al: ${ }^{14}$

$$
\mathrm{MMP}=\left\{\frac{\left[1-\alpha^{\left.\left(\frac{1}{n}\right)\right]} \times\left\{\mathrm{N}-\left[\frac{(\mathrm{n}-1)}{2}\right]\right\}\right.}{(\mathrm{N})}\right\}(\times 100)
$$

\section{Results}

The allelic frequency of the mutant allele was $61.2 \%$ in Collies, $15.6 \%$ in Australian Shepherds, and $7.9 \%$ in Shetland
Sheepdogs $(95 \%$ CI $=55 \%-68 \%, 3 \%-28 \%$, and $4 \%-12 \%$ respectively); and $0 \%$ in Border Collies, German Shepherds, Whippets, and Old English Sheepdogs (MMP $=3.8 \%, 5.2 \%$, $5.4 \%$, and $13.8 \%$, respectively). The MDR1 (-/-) genotype was present in $35.9 \%$ of Collies ( $95 \% \mathrm{CI}=26.9 \%-45 \%$ ), and the MDR1 (+/-) genotype was present in $50.5 \%$ Collies, 31.3\% Australian Shepherds, and 15.8\% Shetland Sheepdogs $(95 \% \mathrm{CI}=26.9 \%-45 \%, 41.1 \%-59.9 \%$, and $7.7 \%-23.9 \%$ respectively) (Table 1).

An analysis of the 181 pedigrees was performed which suggested considerable inbreeding, linebreeding, and also imported lineage (Table 2). Most animals were imported or had parentage imported from European countries and the US. Tables 3 and 4 show the distribution of MDR1 genotypes according to breeding type in the Collie and Shetland Sheepdog breeds, respectively. Although many animals originated from different Brazilian states, the vast majority had the same ancestors in the pedigree. No other correlations were found.

\section{Discussion}

While the total number of individuals sampled for each breed in this survey was determined based on the number of registered animals in the CBKC (eg, Old English Sheepdog, Whippet, Australian Shepherd, and Shetland Sheepdogs), the sample number and selection was influenced by the willingness of the owners to participate in the study. A high number of Border Collie, Australian Shepherd, and Shetland Sheepdog breeders were reluctant or unwilling to participate in the study, therefore there may have been some unintended selection bias. These same difficulties, however, have been reported in other studies from the US, UK, Japan, France, Germany, and Australia. ${ }^{7,13,15-19}$

Of all the breeds surveyed, the MDR1 nt230 (de14) mutated allele was detected only in the Australian Shepherd, Collie, and Shetland sheepdog breeds. In Australian Shepherds, the mutant allele was detected at a frequency of $15.6 \%$

Table I Results of MDRI genotyping with the genotype and allele mutant frequency for each studied breed

\begin{tabular}{|c|c|c|c|c|c|c|c|}
\hline \multirow[t]{2}{*}{ Breed $(\mathbf{N})$} & \multicolumn{2}{|c|}{ MDRI (-I-) } & \multicolumn{2}{|c|}{ MDRI (+/-) } & \multicolumn{2}{|c|}{ MDRI (+/+) } & \multirow{2}{*}{$\begin{array}{l}\text { Mutant allelic } \\
\text { frequency (\%) }\end{array}$} \\
\hline & $\mathbf{n}$ & $\%$ genotype & $\mathbf{n}$ & \% genotype & $\bar{n}$ & $\%$ genotype & \\
\hline Australian Shepherd (16) & 0 & 0 & 5 & 31.3 & 11 & 68.2 & 15.6 \\
\hline Collie (I03) & 37 & 35.9 & 52 & 50.5 & 14 & 13.6 & 61.2 \\
\hline Border Collie (77) & 0 & 0 & 0 & 0 & 77 & 100.0 & 0 \\
\hline German Shepherd (55) & 0 & 0 & 0 & 0 & 55 & 100.0 & 0 \\
\hline Old English Sheepdog (20) & 0 & 0 & 0 & 0 & 20 & 100.0 & 0 \\
\hline Shetland Sheepdog (76) & 0 & 0 & 12 & 15.8 & 64 & 84.2 & 7.9 \\
\hline Whippet (53) & 0 & 0 & 0 & 0 & 53 & 100.0 & 0 \\
\hline
\end{tabular}

Abbreviations: MDRI (-l-), genotype with two mutant alleles, homozygous for the deletion; MDRI (+l-), genotype with one wild type and one mutant allele, heterozygous for the deletion; MDRI $(+/+)$, genotype with two wild type alleles, homozygous; N, number of studied animals for each breed; n, number of animals of each genotype. 
Table 2 Analysis of the 181 pedigrees

\begin{tabular}{|c|c|c|c|c|c|c|c|}
\hline Breed & $\begin{array}{l}\text { Pedigree } \\
\text { available }\end{array}$ & $\begin{array}{l}\text { Pedigree } \\
\text { not available }\end{array}$ & $\begin{array}{l}\text { Imported } \\
\text { strain }^{\mathbf{a}}\end{array}$ & $\begin{array}{l}\text { National } \\
\text { strain }^{\mathrm{a}}\end{array}$ & Outbreeding & Inbreeding & Linebreeding \\
\hline Australian Shepherd & 4 & 12 & 3 & 1 & 4 & 0 & 0 \\
\hline Collie & 64 & 39 & 23 & 41 & 36 & 6 & 22 \\
\hline Border Collie & 34 & 43 & I & 33 & 28 & 0 & 6 \\
\hline German Shepherd & 3 & 52 & 0 & 3 & 3 & 0 & 0 \\
\hline Old English Sheepdog & 12 & 8 & 2 & 10 & 8 & $\mathrm{I}$ & 3 \\
\hline Shetland Sheepdog & 46 & 30 & 18 & 28 & 31 & 1 & 14 \\
\hline Whippet & 18 & 35 & 4 & 14 & 13 & 0 & 5 \\
\hline
\end{tabular}

Note: animal was imported or had imported parentage.

(95\% CI $=3.1 \%-28.2 \%)$. This is likely similar to what was found in the US. ${ }^{7}$ No homozygous mutant genotypes were detected in this breed, and this finding is likely similar to the results of a study conducted in Germany by Baars et al. ${ }^{13}$ However, the use of small sample size in the current study and in the study by Baars et $\mathrm{al}^{13}$ cannot help us infer the absence of this genotype, and its importance in Brazil. The prevalence of the heterozygous genotype $(31.3 \%, 95 \% \mathrm{CI}=26.9 \%-45 \%)$ was inferred to be similar to that observed in Slovenia (30\%), ${ }^{20}$ the US $(29.8 \%),{ }^{7}$ and Germany $(25.2 \%),{ }^{9}$ but is likely to be lower than that observed by others in the US (37\%), ${ }^{11}$ Australia $(42.8 \%),{ }^{16}$ and UK (42.9\%). ${ }^{19}$ In this survey, the confidence intervals for the heterozygous genotype $( \pm 22.7 \%)$ and the allele mutant $( \pm 12.5 \%)$ for this breed are quite wide, thus our ability to make inferences concerning the prevalence, or relative prevalence, of the genotype or mutation is limited. These limitations, however, do not negate the importance of these findings, as they reinforce the concern that individuals of this breed in Brazil may be at risk for clinical toxicosis and adverse or unexpected drug reactions. ${ }^{21}$

In the Collies sampled, allelic frequency for the mutant MDR1 allele was 61.2\% (95\% CI $=54.8 \%-67.5 \%)$, suggesting that the MDR1 mutation associated with multidrug sensitivity is broadly dispersed in Collies living in Brazil. This is likely similar to findings in Germany $(59 \%)^{12}$ and Japan $(58.4 \%)^{18}$ but higher than findings in Belgium $(57.2 \%)^{22}$ and

Table 3 Relationship between the breeding types and MDRI genotype in Collies

\begin{tabular}{llll}
\hline MDR I & \multicolumn{2}{l}{ Pedigree Collie } & \\
\cline { 2 - 4 } genotype $^{a}$ & Outbreeding & Inbreeding & Linebreeding \\
\hline MDRI (-I-) & I4 & 4 & 3 \\
MDRI (+/-) & 17 & 2 & 18 \\
MDRI (+/+) & 5 & 0 & I \\
\hline
\end{tabular}

Note: aNumber of animals found in each breeding type.

Abbreviations: MDRI (-l-), genotype with two mutant alleles, homozygous for the deletion; MDRI $(+/-)$, genotype with one wild type and one mutant allele, heterozygous for the deletion; MDRI $(+/+)$, genotype with two wild type alleles, homozygous. the US $(54.6 \%-56.4 \%),{ }^{7,11}$ and likely lower than findings in the UK $(72.6 \%)^{19}$ and France $(64 \%) .{ }^{17}$ These results are consistent with the current breeding and the importation practices reported in Brazil (Tables 2 and 3). The Collie was the only breed surveyed in which the homozygous mutant genotype was detected. The frequency at which the heterozygous mutant was detected in this survey was, as expected, higher than that of the homozygous mutant, and this genotype can be inferred to be more prevalent in the Brazilian Collie population, as observed in some global studies, ${ }^{7}$ and other specific studies from Germany, ${ }^{9,12}$ but different from those in which there was a higher frequency of the homozygous mutant condition reported. ${ }^{17-19}$ Unlike the findings in the Australian Shepherd, the confidence intervals here for both the homozygous $( \pm 9.1 \%)$ and heterozygous $( \pm 9.4 \%)$ genotypes and the allele mutant $( \pm 6.3 \%)$ are relatively narrow. This increases the power of these conclusions and supports our inference that the prevalence of this mutation is high in the Brazilian Collie population. This also emphasizes the importance of the mutation in this breed and is consistent with the high frequency presented in the scientific literature. Unlike the pedigree analysis conducted in the northwestern United States, ${ }^{15}$ dogs from different Brazilian states were related within the four most recent generations. This finding likely reflects the small number of show breeders in Brazil, and also the frequent use of the same champions for breeding.

Table 4 Relationship between the breeding types and MDRI genotype in Shetland Sheepdogs

\begin{tabular}{llll}
\hline MDR I & \multicolumn{2}{l}{ Pedigree Shelties } \\
\cline { 2 - 4 } genotype $^{\mathrm{a}}$ & Outbreeding & Inbreeding & Linebreeding \\
\hline MDRI (-l-) & 0 & 0 & 0 \\
MDRI (+/-) & 2 & $\mathrm{I}$ & 2 \\
MDRI $(+/+)$ & 29 & 0 & 12 \\
\hline
\end{tabular}

Note: aNumber of animals found in each situation.

Abbreviations: MDRI (-l-), genotype with two mutant alleles, homozygous for the deletion; MDRI (+l-), genotype with one wild type and one mutant allele, heterozygous for the deletion; MDRI $(+/+)$, genotype with two wild type alleles, homozygous. 
In the Shetland Sheepdogs sampled in Brazil, 7.9\% (95\% $\mathrm{CI}=3.7 \%-12.1 \%$ ) had at least one mutant MDR1 allele. This frequency is similar to reports in the US at $6.6 \%{ }^{11}$ to $8.4 \%$, and may be similar to Japan at $1.2 \% \cdot{ }^{18} \mathrm{~A}$ higher frequency of the MDR1 mutant allele was detected in Germany at $30 \% 0^{9,12}$ and in the UK at $35.7 \% .{ }^{19}$ In this breed, as in the Australian Shepherd, our survey detected no homozygous mutants, as observed in Australia ${ }^{16}$ and Japan. ${ }^{18}$ However, the limited sample sizes in this study do not allow for inference of low prevalence of MDR1 mutation. As with the Collie breed, the relatively narrow confidence intervals for the heterozygous genotype $( \pm 8.1 \%)$ and the allele mutant $( \pm 4.2 \%)$ strengthens our conclusions. From these findings we can infer that the prevalence of the homozygous mutant genotype is low in Shetland Sheepdogs in Brazil, and that the prevalence of the mutant allele likely mirrors that of the US. This is consistent with the fact that this breed is largely imported from the US (Tables 2 and 4).

In this survey, no mutant MDR1 alleles were detected in Border Collies or brown colored German Shepherds, and this is comparable to findings from Australia, Belgium, and Germany. ${ }^{12,16,22}$ The same was observed for Old English Sheepdog and Whippets. While our survey failed to detect allelic mutations in these breeds, these findings do not exclude the possibility that the mutation is present in these populations. As such, MMP with $95 \%$ CI was calculated for the MDR1 mutant allele for each of these breeds in Brazil (including heterozygous and homozygous), and was found to be $13.8 \%$ for Old English Sheepdog population in Brazil. This is similar to the findings in the US ${ }^{7,11}$ in which the prevalence of mutant genotypes ranged from $2.5 \%$ to $7.3 \%$ and in Europe (eg, Germany ${ }^{9}$ and the UK ${ }^{19}$ ), from $2.5 \%$ to $21.2 \%$. This finding is not unexpected as many Brazilian Old English Sheepdogs are imported from Europe and the US.

The same observations can be made for Border Collies, German Shepherds, and Whippets, in which the maximum detectable prevalence was $3.8 \%, 5.2 \%$, and $5.4 \%$, respectively. For the Border Collies, the observed prevalence ranges from $0 \%$ to $1.6 \%$ in the US, ${ }^{7,11}$ from $0 \%$ to $4.7 \%$ in the UK, ${ }^{19}$ and from $0.6 \%$ to $1 \%$ in Germany. ${ }^{9,12}$ From this we can infer that the prevalence of the mutation is relatively low in the Brazilian Border Collie population, and likely other global studies. The same is observed for the Brazilian German Shepherd. The MMP found in Brazil was 5.2\%, whereas in US the prevalence ranges from $0 \%$ to $10.2 \%$. ${ }^{7,11}$ However, as concluded by Erkens et al, ${ }^{22}$ we cannot infer the absence of this mutation in the Brazilian German Shepherd, since this breed is usually associated with the Swiss White
Shepherd, which has an observed prevalence ranging from $10 \%$ to $14 \% .^{10-12}$ The possible low prevalence of MDR 1 mutation in Brazilian German Shepherds can be justified by the fact that this breed is an independent breed line from the Swiss White Shepherd in the same way as in Germany, but differently from the US.

For Whippet, the maximum expected prevalence in Brazil is 5.4\%, whereas in the US this ranges from $58.3 \%$ to $76.4 \%$. ${ }^{711}$ This discrepant result might be explained by the fact that in Brazil the Whippet is considered an unacknowledged breed and is usually referred to as Whippet, which until now is considered free of this mutation, and most of our samples included animals with national strain. Moreover, the breeding of Whippet is narrowed to only few registered breeders in the CBKC. This study included 42 samples from two different breeders and 11 samples from random dogs, so most of them had a similar strain which could have resulted in unintended selection bias. Thus, an extended study analyzing different types of strains is necessary to infer the absence and importance of this mutation in the Brazilian Whippets.

The MDR1 mutation by homozygosis or heterozygosis was observed in inbreeding (father to daughter, half-brother to half-sister) and linebreeding (granddaughter to grandsire) practices for Collies (Table 3) and Shetland Sheepdogs (Table 4). In this case, inbreeding results were far less frequent than linebreeding or outbreeding.

Genotype-based selection can eliminate the mutation from the gene pool of affected dog breeds in a few generations in dependence of the MDR1 mutant allele frequency. The design of breeding strategies has to regard the MDR1 mutant allele frequency in the particular dog breed and the population size because the effective population size is reduced and inbreeding is increased with the proportion of heterozygote carriers excluded from breeding. The number of matings per sire has to be limited, and the percentage of heterozygote by homozygote matings [MDR1 (+/-) X MDR1 $(+/+)]$ has to be increased for a limited number of generations. In breeds with high frequency of the mutated allele (eg, Collies), heterozygous and homozygous carriers cannot be immediately excluded. Therefore, the result of gene test for the MDR1 mutant allele should be taken into account when puppies are preselected for breeding. Employing the gene test at this early stage is useful to reduce the MDR1 mutant allele in the candidates of later breeding animals. ${ }^{23}$

The main reason for the genetic selection of these animals is to reduce the therapeutic risks they are subject to. Once the mutation produces a truncated P-gp, a membrane transporter responsible for cell protection against a variety of substances, 
mutant homozygous and heterozygous animals are usually susceptible to P-gp substrates. ${ }^{8}$ P-gp substrates include a vast number of drugs, many commonly used in veterinary medicine, ${ }^{24,25}$ such as chemotherapeutic drugs, immunosuppressants, antiparasitic drugs (mainl ivermectin, moxidectin), steroid hormones, antibiotics, analgesics, antidiarrheals, antiepileptic agents, cardiac drugs, and others..$^{25,26}$ The most important clinical consequence is the neurotoxic effect promoted by the dysfunction of P-gp, which shows clinical signs such as mydriasis, stupor, lethargy, vomiting, ataxia, tremors, drooling, coma, blindness, convulsion, and even death. ${ }^{10,27}$ Therefore, a genetic program would help decrease the number of susceptible dogs and also help veterinarians select a better treatment protocol for the diagnosed mutant patients.

No surveys of the MDR1 mutation in Brazilian breeds have been previously published for reference, and screening for the mutation is not routinely practiced. The dog breeds being discussed are maintained through endogamic crossings. Crosses between heterozygotes and homozygotes will increase the frequency of the MDR 1 mutant allele in the population and crosses between homozygotes will dramatically amplify the frequency of the mutation. As most of these studied breeds were imported from the US and Europe, this study suggests a founder effect ${ }^{7}$ for these Brazilian animals, but with no serious consequence due the equilibrium with the endogamic crossings, population admixture and the crossing politics, resulting in no drastic difference observed from the US and European prevalence.

Breeding strategies should aim to confine or eliminate the MDR1 mutant allele. The progress toward MDR1 mutant allele-free populations is strongly dependent on its prevalence, the population size, and possible correlated adverse effects of the MDR1 allele. In practical terms, considering the frequency of the mutation in each breed sample and the inferred or expected prevalence in Brazil as compared to the US and Europe populations, similar precautions should be taken in the screening of breeding animals and individuals receiving preventative or therapeutic drug intervention.

\section{Conclusion}

Although only Collies were found with the homozygous mutation, Australian Shepherd and Shetland Sheepdog carried at least one mutated allele in Brazil. In the studied samples, the MDR1 mutant allele was not found in Border Collies, Whippets, Old English Sheepdogs, or German Shepherds. Thus, it can be inferred that there is a low occurrence of this mutated allele in Brazilian dogs, and that there is either very low prevalence of the mutated genotype in these populations or it is absent. Due to the important role of $\mathrm{P}$-gp in blood-brain barrier integrity, drug pharmacokinetics and pharmacodynamics, and the serious clinical consequences of adverse drug effects in P-gp deficient individuals, this information is important not only for MDR1 genotypebased breeding programs and the international exchange of breeding animals of predisposed breeds, but also for the modification of drug therapy and availability of genotyping tests for breeds at risk in Brazil.

\section{Acknowledgments}

The authors thank the veterinarians who contributed by providing blood samples and case information about this patient and Prof Dr Ligia Souza Lima Silveira da Mota for all the support provided for the genetic work.

\section{Author contributions}

CB and JPAJ conceived the study. MMM and RCS participated in blood sampling and built the database. MMM ran the genetic tests at JPAJ's laboratory, Unesp Botucatu - Brazil. RCS performed the statistical analysis. All authors participated in data interpretation. Manuscript was written by MMM and CB, reviewed and edited by JPAJ, RCS, and KVL. All authors read and approved the final manuscript.

\section{Disclosure}

The authors report no conflicts of interest in this work.

\section{References}

1. Dean M, Rzhetsky A, Allikmets R. The human ATP-binding cassette (ABC) transporter superfamily. Genome Res. 2001;11:1156-1166.

2. Thiebaut F, Tsuruo T, Hamada H, et al. Cellular localization of the multidrug-resistance gene product P-glycoprotein in normal human tissues. Proc Natl Acad Sci U SA. 1987;84:7735-7738.

3. Cordon-Cardo C, O’Brien JP, Boccia J, et al. Expression of the multidrug resistance gene product (P-glycoprotein) in human normal and tumor tissues. J Histochem Cytochem. 1990;38:1277-1287.

4. Chaudhary PM, Roninson IB. Expression and activity of P-glycoprotein, a multidrug efflux pump, in human hematopoietic stem cells. Cell. 1991;66:85-94.

5. Mealey KL, Bentjen SA, Gay JM, et al. Ivermectin sensitivity in collies is associated with a deletion mutation of the mdr1 gene. Pharmacogenetics. 2001;11:727-733.

6. Roulet A, Puel O, Gesta S, et al. MDR1-deficient genotype in Collie dogs hypersensitive to the P-glycoprotein substrate ivermectin. Eur $J$ Pharmacol. 2003;460:85-91.

7. Neff MW, Robertson KR, Wong AK, et al. Breed distribution and history of canine mdr1-1Delta, a pharmacogenetic mutation that marks the emergence of breeds from the collie lineage. Proc Natl Acad Sci USA. 2004;101:11725-11730.

8. Dowling P. Pharmacogenetics: it's not just about ivermectin in collies. Can Vet J. 2006; 47:1165-1168.

9. Geyer J, Doring B, Godoy JR, et al. Frequency of the nt230 (del4) MDR1 mutation in Collies and related dog breeds in Germany. J Vet Pharmacol Ther. 2005;28:545-551. 
10. Geyer J, Klintzsch S, Meerkamp K, et al. Detection of the nt230(de14) MDR1 mutation in White Swiss Shepherd dogs: case reports of doramectin toxicosis, breed predisposition, and microsatellite analysis. J Vet Pharmacol Ther. 2007;30:482-485.

11. Mealey KL, Meurs KM. Breed distribution of the ABCB1-1Delta (multidrug sensitivity) polymorphism among dogs undergoing ABCB1 genotyping. J Am Vet Med Assoc. 2008;233:921-924.

12. Gramer I, Leidolf R, Doring B, et al. Breed distribution of the nt230(del4) MDR1 mutation in dogs. Vet J. 2011;189:67-71.

13. Baars C, Leeb T, von Klopmann T, et al. Allele-specific polymerase chain reaction diagnostic test for the functional MDR1 polymorphism in dogs. Vet J. 2008;177:394-397.

14. Cannon RM, Roe RT. Australian Bureau of Rural Science. Livestock Disease Surveys: A Field Manual for Veterinarians. Canberra: Australian Government Publishing Service; 1982.

15. Mealey KL, Bentjen SA, Waiting DK. Frequency of the mutant MDR1 allele associated with ivermectin sensitivity in a sample population of collies from the northwestern United States. Am J Vet Res 2002;63:479-481.

16. Mealey KL, Munyard KA, Bentjen SA. Frequency of the mutant MDR1 allele associated with multidrug sensitivity in a sample of herding breed dogs living in Australia. Vet Parasitol. 2005;131:193-196.

17. Hugnet C, Bentjen SA, Mealey KL. Frequency of the mutant MDR1 allele associated with multidrug sensitivity in a sample of collies from France. J Vet Pharmacol Ther. 2004;27:227-229.

18. Kawabata A, Momoi Y, Inoue-Murayama M, et al. Canine mdr1 gene mutation in Japan. JVet Med Sci. 2005;67:1103-1107.
19. Tappin SW, Goodfellow MR, Peters IR, et al. Frequency of the mutant MDR1 allele in dogs in the UK. Vet Rec. 2012;171:72.

20. Cotman M, Zabavnik J. Mutation of MDR1 gene associated with multidrug sensitivity in Australian Shepherds in Slovenia. Slov Vet Res. 2007;44:19-24.

21. Mealey KL, Northrup NC, Bentjen SA. Increased toxicity of P-glycoprotein-substrate chemotherapeutic agents in a dog with the MDR1 deletion mutation associated with ivermectin sensitivity. J Am Vet Med Assoc. 2003;223:1453-1455.

22. Erkens T, Daminet S, Rogiers C, et al. Presence of the ABCB1 (MDR1) deletion mutation causing ivermectin hypersensitivity in certain dog breeds in Belgium. Vlaams Diergeneesk Tijds. 2009;78:256-260.

23. Fecht S, Distl O. Review of prevalence, genetic aspects and adverse effects of the mdr1-1Delta mutation in dogs. Dtsch Tierarztl Wochenschr. 2008;115:212-219.

24. Fromm MF. Importance of P-glycoprotein at blood-tissue barriers. Trends Pharmacol Sci. 2004;25:423-429.

25. Mealey KL. Therapeutic implications of the MDR-1 gene. $J$ Vet Pharmacol Ther. 2004;27:257-264.

26. Martinez M, Modric S, Sharkey M, et al. The pharmacogenomics of P-glycoprotein and its role in veterinary medicine. $J$ Vet Pharmacol Ther. 2008;31:285-300.

27. Seward RL. Reactions in dogs given ivermectin. J Am Vet Med Assoc. 1983;183:493.
Veterinary Medicine: Research and Reports

\section{Publish your work in this journal}

Veterinary Medicine: Research and Reports is an international, peer-reviewed, open access journal publishing original research, case reports, editorials, reviews and commentaries on all areas of veterinary medicine. The manuscript management system is completely online and includes a very quick and fair peer-review system.

\section{Dovepress}

Visit http://www.dovepress.com/testimonials.php to read real quotes from published authors. 